Published in Applied Economics, 2020, vol. 52, no. 31, pp. 3337-3353,

\title{
A rank-dependent utility approach to model intra- and inter-individual heterogeneity in risky choice behaviors
}

\author{
Hélène Bouscasse $^{*} \quad$ Matthieu de Lapparent ${ }^{\dagger}$
}

\begin{abstract}
Using a stated preferences survey, the objective of this paper is to investigate the intra- and inter-individual heterogeneity of mode choice, when travel time is subject to variability. By"inter-individual heterogeneity" is meant that people are different in terms of attitude toward risk and have different functional forms for the utility function. By "intra-individual heterogeneity" is meant that the behavior may be different even when performed by the same individual when faced with a different transport mode. Based on Rank-Dependent Utility Theory, the paper shows that the probabilities of delay associated with train trips are overweighted and the probabilities associated with car trips are underweighted. A latent-class model offers a somewhat different view: if, overall, car users do indeed weight the probabilities for train and car trips differently, the train users seem to be risk neutral and consider the objective probabilities that are presented in the stated preferences survey.
\end{abstract}

\section{Introduction}

All travelers face travel time uncertainty whatever their transport mode. However, the perception of travel time varies depending on travelers and transport modes, which leads to inter- and intra-individual heterogeneity. A strong hypothesis of the economic theory is that preferences are stable over time and context, which controverts intra-individual heterogeneity. However, behavioral experiments show evidence of context-dependent preferences (Mazar et al., 2014; Tversky and Simonson, 1993). The objective of this paper is to model the two types of heterogeneity by integrating Rank-Dependent Utility Theory (RDUT) in a random utility model to explain mode choice. Inter-individual heterogeneity is taken into account by means of a latent-class model, while intra-individual heterogeneity is considered using probability weightings that depend on travel mode. In doing so, this paper also responds to the criticism of Rasouli and Timmermans (2014) regarding the lack of work on "contextdependent heterogeneity and taste differences among travelers".

\footnotetext{
${ }^{*}$ Corresponding author: Grenoble Applied Economics Laboratory, University Grenoble Alpes, CNRS, 1241 rue des résidences, 38400 Saint-Martin-d'Hères, France. helene.bouscasse@univ-grenoble-alpes.fr

${ }^{\dagger}$ School of Engineering and Management Vaud (HEIG-VD). University of Applied Sciences and Arts Western Switzerland (HES-SO). Avenue des Sports, 20. CH-1401 Yverdon-les-Bains. Switzerland. matthieu.delapparent@heig-vd.ch
} 
Among the wide body of literature on travel time variability, only a few studies are concerned with mode choice (Bhat and Sardesai, 2006; König and Axhausen, 2002; Batarce and Ivaldi, 2014; de Lapparent and Ben-Akiva, 2014; Hensher et al., 2015). Moreover, most of them have been developed within a linear utility framework. The mean-variance and scheduling models, typically applied with stated preferences data, are the two dominant approaches to empirical measurement of the value of travel time variability (e.g., Börjesson et al., 2012; Bates et al., 2001).11 These approaches make two strong assumptions: people are risk neutral and they consider objective probabilities in their decisions. Such assumptions have been challenged by the economic theory, and transportation research started to incorporate theories such as Rank-Dependent Utility Theory (RDUT; Quiggin, 1982; Yaari, 1987) or (cumulative) prospect theory (Kahneman and Tversky, 1979; Tversky and Kahneman, $1992)$ a decade ago. de Palma et al. (2008) provide a general overview of choice analysis for both EUT and non-EUT approaches in the context of modeling travelers' risky choices and, in an editorial, de Palma et al. (2015) review various studies on risk and uncertainty in transport and urban economics.

Li and Hensher (2011) conduct a literature review on the use of (cumulative) prospect theory in different fields of transport: route choice, departure time choice, parents' choice of picking up their child and network equilibrium. They notice that most works only draw selectively on some contributions from prospect theory and highlight the absence of willingnessto-pay estimates and consideration of unobserved between-individual heterogeneity. As an answer, Hensher et al. (2011) estimate logit and mixed logit models which incorporate probability weighting and risk attitude in a route choice context and derive estimates of Value Of Time (VOT). Hensher and Li (2012) propose a similar work using RDUT. Razo and Gao (2013) specify a latent-class model which incorporates RDUT to explain strategic route choice under real-time traffic information. Jou and Chen (2013) apply cumulative prospect theory to freeway drivers' route choice behaviors. They study behavioral heterogeneity by integrated individual variables to explain the parameters of the model (risk aversion, loss aversion, probability weightings). Xiao and Fukuda (2015) reformulate a general scheduling model employing rank-dependent utility theory and study the uncaptured costs of travel time variability. To my knowledge, there are only two applications of RDUT to mode choice. The first is de Lapparent and Ben-Akiva (2014) who compare different parametric forms to analyze risk attitudes and probability weightings. They conclude that commuters are weakly averse to small-time losses and that leisure travelers are risk neutral to small losses of time. However, in their data, only probabilities vary and not the delay times. The second is Hensher et al. (2015) who conduct a survey in which they ask the respondents to report three perceived travel times for car and public transport, as well as the probability associated with each travel time. They conclude that utility does not depend on the source of uncertainty (see Fox and Tversky, 1995; Tversky and Fox, 1995, for an introduction to this concept) and is the same for risk and uncertainty.

Although the literature using probability weighting functions in the field of transportation is growing, parameter estimations from travel behavior are still limited. The

\footnotetext{
${ }^{1}$ For appraisal of transport projects, the former is the only relevant option since it relies on statistical measures of variability (for instance, standard deviation). However, the microeconomic foundations of how travel time variability generates costs are hidden. In contrast, the scheduling model is microeconomically sound.
} 
behavioral economics literature mainly bases its estimations on decision making under risk for monetary outputs. However, there are fundamental differences between simple gambling behavior and complex travel behaviors (Timmermans, 2010; Kemel and Paraschiv, 2013). Firstly, travel behavior is characterized by multiple attributes (travel time but also cost, frequency of public transport, comfort, and so on). Secondly, probabilities are generally unknown such that a decision is made under uncertainty. However, due to the repeated nature of travel decisions, the decision maker experiences the consequences of her decision and therefore acquires some information about the travel time distribution, which also makes travel decisions different from other decisions under uncertainty.

The motivation for analyzing heterogeneity stems from an upstream qualitative analysis. ${ }^{2}$ A first observation from this analysis is that people seem to have different perceptions of travel time uncertainty depending on the travel mode. With regard to train travel, the departure time and the arrival time are written on a schedule. If the train arrives just a few minutes behind schedule, then some people consider that it is late. Other people are more flexible and consider that the train is late when the delay exceeds 15 minutes. The departure and arrival times of car travel, by contrast, are not written down on a schedule. Therefore, delay is only defined relative to the preferred arrival time (for instance, an appointment time). For instance, for a scheduled travel time of 90 minutes, if a train is 15 minutes late, then the traveler will perceive the delay. In the same conditions by car, the final travel time will probably not be categorized as delayed. Trains are therefore perceived as more frequently delayed than cars. A second observation is that, in the event of train delays, most people feel trapped and they have no control over the situation, whereas in the event of car delays, people feel responsible and are active in their car. In some situations, they may even change their route. Delays are therefore experienced more negatively during travel by train than by car, and will therefore be more pregnant in memories (Baumeister et al., 2001). A third observation is that this opposition in perceptions between trains and cars is especially true for car users. Train users seem to be more aware of the travel time distribution for both modes. In other words, the lessons learned from the qualitative phase is that the event "delay" is associated with unknown probabilities and is not accurately but fuzzily measured. To draw a parallel with lotteries, this would correspond to a situation in which the lottery amounts are known with different fuzziness and the probabilities as well.

As there does not appear to be any revealed preferences survey available to analyze heterogeneous behaviors when travel time is uncertain, the analysis is based on a stated preferences survey. The delay time and probabilities are clearly written down and decision makers are put in a situation of risk and not of uncertainty. The two following hypotheses are formulated: 1) people carry their real-life bias into the stated preferences survey, and since they usually misrepresent the delays and probabilities of delay, they will also do so in the survey; 2) the misrepresentation weighs on the probabilities and not on the perception of delays.

The contribution of this paper to the current knowledge on mode choice is fourfold. Firstly, to my knowledge, it is one of the first application of RDUT to mode choice with probabilities and delay times which vary across choice questions. Almost all other applications of RDUT or (cumulative) prospect theory have modeled route choice. In their

\footnotetext{
${ }^{2}$ Two focus groups of 13 persons and 11 individual interviews.
} 
application to mode choice, Hensher et al. (2015) consider probabilities which are stated by the respondent and, in de Lapparent and Ben-Akiva (2014), the delay times are fixed. Secondly, this work is one of the first to study intra-individual heterogeneity, and, more specifically to show how probability weightings depend on the travel mode. Thirdly, this work is one of the first to differentiate risk attitudes in a RDUT framework with a latent class model. Previous studies have modeled behavioral heterogeneity with alternative methods such as mixed logit (de Lapparent and Ben-Akiva, 2014; Hensher and Li, 2012; Hensher et al., 2011) or by allowing behavioral coefficients to depend on individual variables. For instance, by means of an ordered probit model, de Palma and Picard (2005) show that the level of risk aversion is impacted by key socio-economic factors, such as gender, employment status or purpose of the trip. Unlike mixed logit, latent class models "relax [the] requirement that the analyst make specific assumptions about the distributions of parameters among individuals" (Greene and Hensher, 2003, p. 681). It is also computationally less demanding and easier to interpret. To my knowledge, two papers have already used latent-class models to analyze variability in travel time but the class does not define risk attitudes. Razo and Gao (2013) differentiate strategic and non-strategic decisions, strategic decisions referring to the traveler's recognition that certain detours can be taken in response to future information. Sun et al. (2012) define three risk-attitude classes for sequential choices concerning activity rescheduling: the risk avoider considers the worst case at each node, the risk seeker considers the more likely outcome and the risk neutral individual uses expected utility. In their study, there is thus no risk attitude parameter or probability weighting function. Fourthly, studying travel time variability taking into account risk aversion within a RDUT or (cumulative) prospect theory framework also enriches the interpretation of the VOT. Previous works have discussed how VOT increases with the size of the time change (Hjorth and Fosgerau, 2012) or the VOT distribution across the population (Hensher et al., 2011, 2015). To my knowledge, this work is the first to estimate VOTs taking into account a risk aversion parameter in a RDUT framework and to show how it evolves according to travel time.

The rest of the paper is organized as follows. Part 2 presents the main theories of choice under conditions of risk. Part 3 defines the model framework. Part 4 presents the data. Part 5 discusses the results. Part 6 concludes.

\section{Theories of choice under conditions of risk}

Risk-aversion or risk-seeking behaviors are taken into account in Expected Utility Theory (EUT) by means of a transformation $\phi($.$) of the utility function. However, violations of$ EUT, revealed by Allais (1953) and others (see Denant-Boèmont and L'Haridon, 2013, for a review of violations to the EUT), suggest that the "psychological weight attached to an event [...] usually differs from the probability of that event" (Wu and Gonzalez, $1999, \mathrm{p} .74)$.

To account for the perceptual translation of probability $p_{E}$ associated with event $E$, non-linear probability weighting functions $w\left(p^{E}\right)$ have been introduced. The seminal work in this field is Kahneman and Tversky (1979) who developed prospect theory, within which the notion of risk in decision making is represented by a separable function $w\left(p^{E}\right) \times \phi\left(t^{E}\right)$ which defines the value of a prospect that delivers an outcome $t^{E}$ with probability $p^{E}$. In this 
theory, people transform probabilities without taking into account the associated outcome ${ }^{3}$

By contrast, RDUT, suggested by Quiggin (1982) and fully described by Yaari (1987), proposes to determine the weighting function of the probabilities as dependent on the rank of the outcomes (see Diecidue and Wakker, 2001, for an intuitive presentation of RDUT). RDUT turns the valuable concept of probability weighting from the psychological literature into "a theory sound enough for economists to use" (de Palma et al. 2008). Outcomes are ranked from the worst to the best and the probability weights operate in a cumulative way since they depend on the rank of the associated outcome and on the probability. The probability weighting function usually observed in empirical works has an inverse S-shape (e.g., Kahneman and Tversky, 1979; Tversky and Kahneman, 1992; Wu and Gonzalez, 1999), which means an overweighting of small probabilities and underweighting of high probabilities.

\section{Model framework}

\subsection{Embedding RDUT within a random utility model}

For each alternative $j(j=1, \cdots, J)$, consider the prospect $\mathscr{X}_{j}=\left(E_{1}: t_{j}^{E_{1}}, \ldots, E_{S}: t_{j}^{E_{S}}\right)$ with $S$ mutually exclusive events, which occur each with probability $p_{j}^{E_{1}}, \ldots, p_{j}^{E_{S}}$.

The program of the decision maker $n(n=1, \ldots, N)$ is to maximize her utility $U_{n, j}$

$$
U_{n, j}=\sum_{E \in \mathscr{E}} W\left(p_{j}^{E}\right) \tilde{V}_{n, j}\left(\mathbf{x}_{\mathbf{n}}, \tilde{\mathbf{y}}_{\mathbf{n}, \mathbf{j}} \mid E, \tilde{\boldsymbol{\beta}}\right)+\epsilon_{n, j}
$$

with $p_{j}^{E}$ the probabilities associated with each event $E\left(E \in \mathscr{E}=\left(E_{1}, \ldots, E_{S}\right)\right)$ for alternative $j, \tilde{V}_{n, j}$ the deterministic part of the utility which depends on a vector of individual variables $\mathbf{x}_{\mathbf{n}}$ and on a vector of alternative-specific variables $\tilde{\mathbf{y}}_{\mathbf{n}, \mathbf{j}}$, and $\epsilon_{n, j}$ error terms which are assumed to be independently and identically distributed type I Extreme Value across individuals and alternatives: $\epsilon_{n, j} \stackrel{\text { iid }}{\rightarrow} E V(0,1)$. $\tilde{\boldsymbol{\beta}}$ is the parameter vector. For the sake of simplicity, subscripts corresponding to the choice questions are initially omitted.

Under RDUT, the different travel times $t_{E_{s}}(s=1, \ldots, S)$ are ordered such that the least preferred outcome, that is, the longer travel time, is number 1 and the preferred outcome, the shortest travel time, is number $S$. For all $j=1, \cdots, J$, the weighting probability function is

$$
W\left(p_{j}^{E_{s}}\right)=\left\{\begin{array}{lll}
w\left(p_{j}^{E_{s}}\right) & \text { if } \quad s=1 \\
w\left(p_{j}^{E_{1}}+\cdots+p_{j}^{E_{s}}\right)-w\left(p_{j}^{E_{1}}+\cdots+p_{j}^{E_{s-1}}\right) & \text {, if } \quad s=2, \ldots, S,
\end{array}\right.
$$

with $w(0)=0$ and $w(1)=1$.

The hypothesis is formulated that, for each alternative $j$, the travel time $t_{j}$ faced by the decision maker can take two values $(S=2)$ : a travel time with delay $t_{j}^{E_{1}}=\bar{t}_{j}$, which is

\footnotetext{
${ }^{3}$ In prospect theory, formulas "turned out not to be sound because they imply violations of stochastic dominance in preferring less money to more money in manners that are not only normatively but also descriptively unwarranted" (de Palma et al. 2008, p.5).
} 
the worst outcome and occurs with probability $p_{j}^{E_{1}}=p_{j}$; and a travel time with no delay, $t_{j}^{E_{2}}=\underline{t}_{j}$, which is the best outcome and occurs with probability $p_{j}^{E_{2}}=1-p_{j}$. Therefore, the probability weighting function is

$$
W\left(p_{j}\right)=\left\{\begin{array}{lll}
w\left(p_{j}\right) & \text { if } & s=1 \\
1-w\left(p_{j}\right) & \text {,if } & s=2 .
\end{array}\right.
$$

Since the outcome of the events only impacts travel time, the other alternative-specific variables remain unchanged, such that Equation (1) can be re-written

$$
U_{n, j}=f\left(p_{j}, \underline{t}_{j}, \bar{t}_{j}\right)+V_{n, j}\left(\mathbf{x}_{\mathbf{n}}, \mathbf{y}_{\mathbf{n}, \mathbf{j}} \mid \boldsymbol{\beta}\right)+\epsilon_{n, j},
$$

with $\mathbf{y}_{\mathbf{n}, \mathbf{j}}$ the vector of alternative-specific variables except the time, such that the vector $\tilde{\mathbf{y}}_{\mathbf{n}, \mathbf{j}}^{T}=\left(t_{j}, \mathbf{y}_{\mathbf{n}, \mathbf{j}}^{\mathbf{T}}\right), \boldsymbol{\beta}$ the truncated parameter vector such that $\tilde{\boldsymbol{\beta}}^{\boldsymbol{T}}=\left(\boldsymbol{\beta}_{\text {time }}, \boldsymbol{\beta}^{\boldsymbol{T}}\right)$ and $V_{n, j}$ the truncated deterministic part of the utility function.

The risky component of the utility function is

$$
f\left(p_{j}, \underline{t}_{j}, \bar{t}_{j}\right)=\beta_{\text {time }} \times\left[\left(1-w\left(p_{j}\right)\right) \times \phi\left(\underline{t}_{j}\right)+w\left(p_{j}\right) \times \phi\left(\bar{t}_{j}\right)\right],
$$

with $w($.$) and \phi($.$) functions to be defined.$

\subsection{Functional form of $\phi($.}

$\phi($.$) is the value function and expresses the risk aversion of the decision maker. Constant$ relative risk aversion utility functions have been widely used in behavioral economics (e.g., Tversky and Kahneman, 1992; Holt et al., 2002). Specifically, with the following specification for all $t_{j} \in\left\{\underline{t}_{j}, \bar{t}_{j}\right\}$

$$
\phi\left(t_{j}\right)= \begin{cases}\frac{t_{j}^{1-\alpha}}{1-\alpha} & , \text { if } \alpha \geq 0 \text { and } \alpha \neq 1 \\ \ln \left(t_{j}\right) & , \text { if } \alpha=1,\end{cases}
$$

$\alpha$ denotes the coefficient of relative risk aversion.

Under EUT, the risk attitude of a decision maker is completely determined by the curvature of the value function. A concave $\phi($.$) suggests a risk-averse attitude and a convex$ $\phi($.$) suggests a risk-seeking attitude, regardless of the outcome of the probabilities.$

\subsection{Functional form of $w($.}

The decision weight $w($.$) may take different functional forms (see de Lapparent and Ben-$ Akiva, 2014; Rasouli and Timmermans, 2014, for an overview). Given the objectives of the paper, two forms are investigated $\left.\right|^{4}$ Firstly, $w($.$) is modeled by means of a power transfor-$ mation

$$
w\left(p_{j}\right)=p_{j}^{\delta}, \delta>0,
$$

\footnotetext{
${ }^{4}$ The Prelec functional form (Prelec, 1998) has also been tested but it provided inconsistent results, possibly due to the two parameters which have to be estimated with this function. Further research is needed to solve this issue. These three parametric functions - Power, T\&K (or Quiggin) and Prelec - are also the ones considered in (de Palma et al. 2008).
} 
which denotes an overweighting of probabilities if $0<\delta<1$ and an underweighting if $\delta>1$. $\delta$ is therefore expected to be lower than one for train trips to reflect pessimism and greater than one for car trips to reflect optimism.5

Secondly, $w($.$) is modeled by means of the transformation proposed by Tversky and$ Kahneman (1992, T\&K)

$$
w\left(p_{j}\right)=\frac{p_{j}^{\delta}}{\left[p_{j}^{\delta}+\left(1-p_{j}\right)^{\delta}\right]^{1 / \delta}}, \delta>0 .
$$

If $\delta=1$, then $w\left(p_{j}\right)=p_{j}$ and EUT applies. If $\delta>1$, then probabilities are underweighted. If $0<\delta<1$, the decision weight function has an inverted S-shape, which means that outcomes with lower probabilities tend to be overweighted whereas outcomes with higher probabilities tend to be underweighted. Put differently, when the probability of delay $p_{j}$ is below the threshold probability $p^{\star}$, then $w\left(p_{j}\right)>p_{j}$ and $1-w\left(p_{j}\right)<1-p_{j}$. Therefore, if the probability of delay observed in the database is below the estimated threshold, that is, $p_{j}<p^{\star}$, then the travel time with delay weighs more than proportionally compared with the travel time with no delay. This reflects pessimism in perceiving the probability of delay.

The risk aversion attitude has to be interpreted in light of the weighting function form (see Cohen, 2015, for a discussion). For instance, if the dual theory of choice under risk (Yaari, 1987) applies, decision makers are weakly risk averse since the value function is linear in $t$, that is, $\alpha=0$, and the probability of delay is overweighted, that is, $\delta<1$ with the power transformation. If the decision maker is pessimistic enough, she might exhibit weak risk aversion even with a convex value function.

\subsection{Tested hypotheses and models}

Based on the observations made during the qualitative analysis, three hypotheses are formulated:

- H1: Probabilities of delays during travel by car are underweighted since the event "delay" is not well defined;

- H2: Probabilities of delays during travel by train are overweighted since the event "delay" is well defined and leads to negative experiences which are more easily retained than the favorable consequences of the event "no delay";

- H3: The distinction in the weighting of probabilities is more accurate for car users than for train users who are more likely to consider objective probabilities when making their choice.

To test these three hypotheses seven models are estimated. Firstly, six nested logit models are estimated. The two train alternatives (see Section 4) are grouped within a first nest while the car alternative is in a second nest. In the first model, people are supposed to be risk neutral and to consider the expected travel time as stated in the scenarios when making

\footnotetext{
${ }^{5}$ Usually, in the gains domain, the inverse relationship is considered: $\delta<1$ denotes an optimistic attitude and $\delta>1$ a pessimistic attitude.
} 
their choice. The second model fits into EUT, with a relative risk aversion coefficient. The third model fits into RDUT, with a power transformation of the probabilities. The fourth model is the same as the third model but estimates two distinct parameters for $\delta$, that is, $\delta^{c a r}$ and $\delta^{\text {train }}$, to take account of intra-individual heterogeneity. The fifth model fits into RDUT, with a T\&K transformation of the probabilities. The sixth model is the same as the fifth model but estimates two distinct parameters for $\delta$, as in the fourth model.

Secondly, a latent class model (Greene and Hensher, 2003) is specified to take into account inter-individual heterogeneity in risk attitudes, in addition to the intra-individual heterogeneity. Two classes are defined. In the first class, decision makers are risk neutral and base their decision on the expected travel time, as in the first nested logit model. In the second class, decision makers may not be risk neutral and have non-linear decision weights, with a T\&K transformation, as in the sixth nested logit model. For decision maker $n$ belonging to class $k(k=1,2)$, the conditional probability of choosing option $j$ in the choice question $q(q=1, \cdots, Q)$, is

$$
\pi_{n, q, j}^{\{k\}}=\frac{\exp \left(\beta_{\text {time }}^{\{k\}}\left[\left(1-w\left(p_{j}\right)\right) \times \phi\left(\underline{t}_{j}\right)+w\left(p_{j}\right) \times \phi\left(\bar{t}_{j}\right)\right]+V_{n, j}\left(\mathbf{x}_{\mathbf{n}}, \mathbf{y}_{\mathbf{n}, \mathbf{j}} \mid \boldsymbol{\beta}^{\{k\}}\right)\right)}{\sum_{i=1}^{J} \exp \left(\beta_{\text {time }}^{\{k\}}\left[\left(1-w\left(p_{i}\right)\right) \times \phi\left(\underline{t}_{i}\right)+w\left(p_{i}\right) \times \phi\left(\bar{t}_{i}\right)\right]+V_{n, i}\left(\mathbf{x}_{\mathbf{n}}, \mathbf{y}_{\mathbf{n}, \mathbf{i}} \mid \boldsymbol{\beta}^{\{k\}}\right)\right)},
$$

with $\beta_{\text {time }}^{\{k\}}$ the parameter associated with travel time for class $k$ and $\boldsymbol{\beta}^{\{k\}}$ the parameter vector specific to class $k$ associated with the other explanatory variables. $\phi($.$) is defined$ in Equation (6) and $w($.$) is defined in Equations (7) and (8). For class 1, two constraints are$ imposed: $\alpha=0$ and $\delta=1$.

For decision maker $n$, the probability $\tilde{p}_{n}^{\{k\}}$ of belonging to class $k$ is defined by a logit model

$$
\tilde{p}_{n}^{\{k\}}=\frac{\exp \left(\boldsymbol{\theta}^{\{k\} T} \mathbf{x}_{\mathbf{n}}^{\star}\right)}{\sum_{k^{\prime}=1}^{2} \exp \left(\boldsymbol{\theta}^{\left\{k^{\prime}\right\} T} \mathbf{X}_{\mathbf{n}}^{\star}\right)},
$$

where $\mathbf{x}_{\mathbf{n}}^{\star}$ denotes a vector of individual variables which enter the model for class membership and $\boldsymbol{\theta}^{\{k\}}$ is the parameter vector associated with class $k$.

The assumption is made that the answers to the $Q$ questions given by the same decision maker are independent given the class assignment ${ }^{6}$ The contribution of decision maker $n$ to the likelihood is

$$
\pi_{n}^{\mathrm{LC}}=\prod_{q=1}^{Q}\left[\tilde{p}_{n}^{\{1\}} \times \prod_{j=1}^{J}\left(\pi_{n, q, j}^{\{1\}}\right)^{c_{n, q, j}}+\tilde{p}_{n}^{\{2\}} \times \prod_{j=1}^{J}\left(\pi_{n, q, j}^{\{2\}}\right)^{c_{n, q, j}}\right]
$$

with

$$
c_{n, q, j}= \begin{cases}1 & , \text { iif } U_{n, q, j} \geq U_{n, q, i}, \forall i=1, \cdots, J \\ 0 & , \text { otherwise. }\end{cases}
$$

\footnotetext{
${ }^{6}$ To take into account the panel structure of the data, this assumption will be relaxed in a future work in which we intend to estimate the same models with Bayesian statistics.
} 
$U_{n, q, j}$ is the utility function defined in Equation (1) which omitted the index $q$ for the sake of simplicity.

The loglikelihood of all observations over all individuals is

$$
\mathscr{L}(\hat{\beta})=\sum_{n=1}^{N} \ln \pi_{n}^{\mathrm{LC}} .
$$

\section{Data}

Between 2012 and 2015, a large travel survey was conducted among 36,000 inhabitants living in the Rhône-Alpes region (France) with geographic stratified sampling. Using this data set, 19,601 inhabitants who had recently traveled by train were asked to answer a web stated preferences survey. 2,396 volunteer respondents were asked to describe in detail (time, cost, purpose and origin), a trip they made by coach, train or car during the last month within the Rhône-Alpes region. Only respondents living in the Rhône-Alpes region, aged 18 or over, having a car and a driving license and whose trip was made or could have been made by train or coach were asked to answer the choice questions, resulting in 1,215 answers. The feasibility of a modal shift was assessed thanks to a database constructed with the software Musliw (Palmier, 2010) and containing travel time by public transport and car for each origin-destination pair of the region. Due to the low rate of train users in the population, they were oversampled with a face-to-face survey made in regional trains, using the quota sampling method (sex, age, motive, travel time and train line). 1,059 respondents began the questionnaire and 608 finished it. The loss is mainly due to trips which were not made entirely within the Rhône-Alpes region or persons not living in the region. After cleaning the data (i.e. deleting respondents who should not have been interviewed or who gave absurd answers), the answers of 1,788 respondents were retained. Eventually, $54 \%$ of the respondents are car users.

Each respondent had to choose between three travel modes, which were described in terms of travel mode, cost, time, probability and time delay, frequency, clock-face timetable and comfort. To avoid a cognitive burden, attributes describing the journey were split into three exercises. In Exercise 1, on which this paper focuses, the two train alternatives (A and B) and the car alternative vary according to travel cost, regular travel time, delay time, probability of delay, and frequency (only for the two train alternatives; see Figure 1).

Travel cost includes public transport ticket or pass, gasoline, parking cost and toll. Travel time is defined from origin to destination (including access time, egress time, waiting time and in-vehicle time). The delay time has one of the following values: 10,15 or 30 minutes. The delay probability has one of the following values: $5 \%, 10 \%$ or $20 \%$, which reflect what is actually observed for trains in the Rhône-Alpes region. The frequency of trains is one every two hours, one every hour, two every hour and four every hour. To improve the efficiency of the design, a Bayesian efficient design was implemented (Rose and Bliemer, 2007). A priori weights of attributes were taken from the literature and adjusted during the pilot tests. Respondents had to answer to five choices questions in Exercise 1, leading to a database with 8,933 observations, since a few respondents did not answer all five questions. The choice questions are personalized with the data collected for the reference 


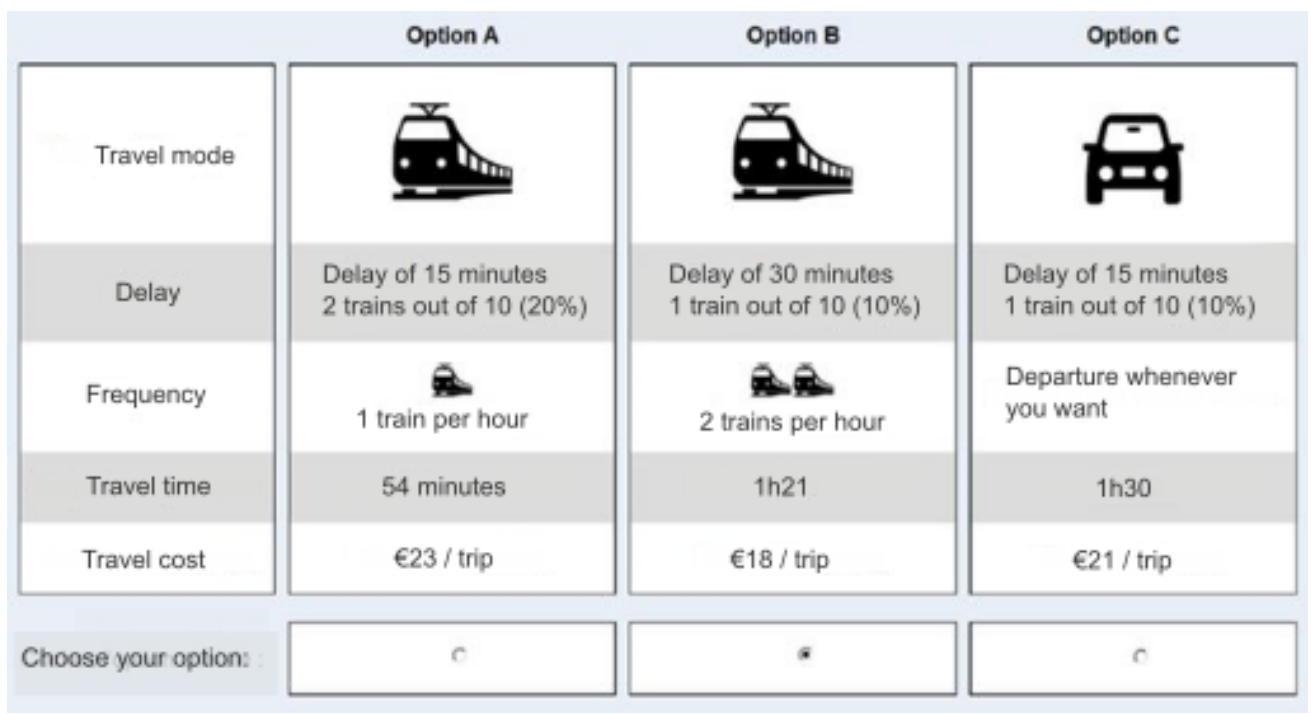

Figure 1 Example of choice question for Exercise 1

journey. In particular, levels of time and cost attributes are pivoted around the values collected for the reference journey. One of the three alternatives was systematically a status quo alternative with the mode, travel time and travel cost identical to the reference journey. The percentage of respondents who chose a train alternative was $47 \%$, while $53 \%$ chose the car alternative. The descriptive statistics of the sample are shown in Table 1 .

Table 1 Descriptive statistics

\begin{tabular}{lrrrrr}
\hline Variable definition & Label & Mean & S.D. & Min & Max \\
\hline Alternative-specific variables & & & & & \\
Travel time by train A (in minutes) & Train time & 67.53 & 51.89 & 5.00 & 325.00 \\
Travel time by train B (in minutes) & Coach time & 67.15 & 52.43 & 5.00 & 375.00 \\
Travel time by car (in minutes) & Car time & 59.07 & 39.04 & 4.00 & 330.00 \\
Travel cost by train A (in $€$ ) & Train cost & 8.57 & 7.28 & 1.00 & 62.00 \\
Travel cost by train B (in $€$ ) & Coach cost & 8.53 & 7.35 & 1.00 & 78.00 \\
Travel cost by car (in $€$ ) & Car cost & 10.34 & 9.17 & 1.00 & 71.00 \\
\hline Individual variables & & & & & \\
Age (in years) & Age & 47.79 & 16.10 & 18.00 & 90.00 \\
Gender (1 if man, 0 if woman) & Gender & 0.50 & & & \\
Car user for the reference trip (1 if yes, 0 & Type_car & 0.54 & & & \\
otherwise) & & & & & \\
Trip's purpose is obligatory (work or study) (1 & Obligatory & 0.42 & & & \\
if yes, 0 otherwise) & & & & &
\end{tabular}




\section{Results}

The estimation of the models uses the software Pythonbiogeme (Bierlaire, 2016). Table 2 shows the estimation results. The car alternative is the reference alternative for the mode choice model and the first class is the reference class for the class membership model. In all models, the car alternative is chosen as the reference alternative. 
Table 2 Estimation results for the seven models

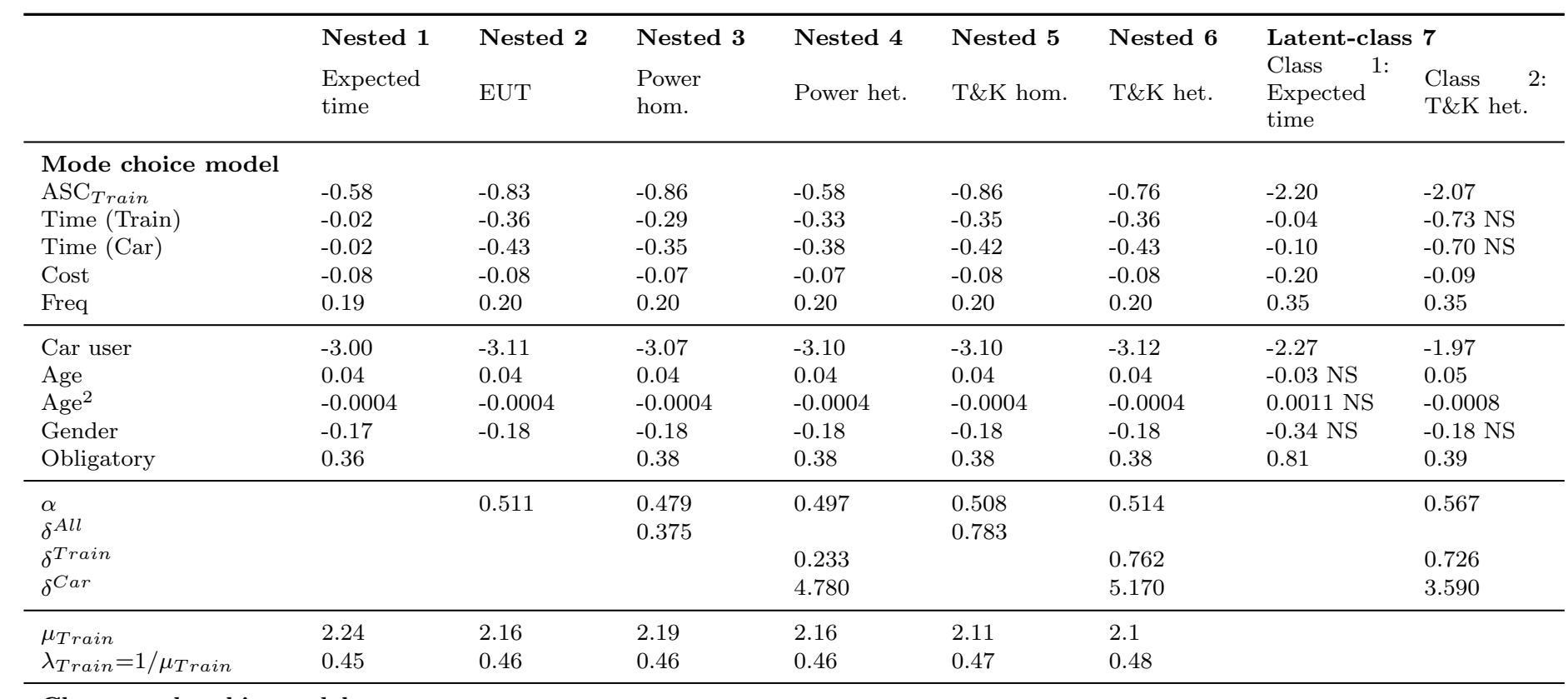

Class membership model

ASC

$-1.01$

Goodness of fit

\begin{tabular}{llllllll} 
Loglikelihood & $-6,641$ & $-6,607$ & $-6,607$ & $-6,601$ & $-6,616$ & $-6,614$ & $-6,540$ \\
AIC & 13,304 & 13,237 & 13,240 & 13,230 & 13,257 & 13,257 & 13,130 \\
$\overline{\rho^{2}}$ & 0.322 & 0.326 & 0.325 & 0.326 & 0.325 & 0.325 & 0.331 \\
Rank & 5 & 3 & 3 & 2 & 4 & 4 & 1 \\
\hline
\end{tabular}

Notes: ASC: Alternative-Specific Constant; hom.=homogeneous; het.=heterogeneous; AIC: Akaike Information Criterion. EUT: Expected Utility Theory; Power: Power probability weighting function; T\&K: Tversky and Kahneman probability weighting function. All variables are significant at the $0.1 \%$ level, except the one indicated as Non-Significant (NS). The $\delta$ parameters are all significantly different from one at the $0.1 \%$ level. The ranking is calculated with the Ben-Akiva and Swait (1986) test. 


\subsection{Goodness of fit}

The adjusted McFadden $\rho^{2}\left(\bar{\rho}^{2}\right)$ and the Akaike Information Criterion (AIC) are used to rank the models in terms of goodness of fit. $]^{7}$ The models being non-nested, one solution is to follow the method proposed by Ben-Akiva and Swait (1986), which determines the asymptotic upper bound of the probability that the difference between the $\bar{\rho}^{2}$ is greater than the observed one. 8 The difference between $\bar{\rho}_{2}$ is sufficient to conclude that the latent class model outperforms all other models, therefore showing the importance of taking into account intra- and inter-individual heterogeneity. Then comes the nested model with a power transformation of travel time and heterogeneous weighting parameters. Third is the EUT nested model and the nested model with a power transformation model and homogeneous weighting parameters. Both are similar in terms of data fit. The two nested models with the $\mathrm{T} \& \mathrm{~K}$ transformation are also similar. The worst ranking model is the nested model, which considers the expected travel time.

\subsection{Estimation results}

The parameter estimates for the nested logit models are robust across specifications and consistent with expectations: all coefficients are significant, with the expected sign. For instance, for Model 1, the probability of choosing an option decreases as travel time and travel cost increase. The higher the frequency of trains, the higher the probability of choosing a train alternative. As age increases, the train alternatives become more attractive but with a decreasing marginal effect. Women and people who travel for work or studies are more likely to choose the train as a transport mode. Moreover, the value $\left(1-\lambda_{\text {Train }}\right)$, which can be used as a measure for correlation (Train, 2009), shows that the two train alternatives are not independent. The nested structure is therefore well adapted to the data.

For Models 2 to 7 , the relative risk aversion coefficients are positive, significantly different from zero and comprised in a narrow range, between 0.479 and 0.567 , which show the robustness of the results across the models. Since the risk refers to a "loss" of time, a positive coefficient is associated with a convex utility function (see Figure 2). For instance, people are willing to pay less to save 10 minutes on a 30-minute trip than on a 60 -minute trip 9 . These coefficients are consistent with the literature. Indeed, the risk aversion coefficients reviewed in $\mathrm{Li}$ and Hensher (2011) are comprised between 0.26 and 0.89 (and even 2.07 in Senbil and Kitamura, 2005). Hensher and Li (2012) estimate a relative risk aversion coefficient equal to 0.29 with a logit model and 0.36 with a mixed logit model. Hensher and Li $(2012)$ interpret these coefficients as denoting risk-seeking attitudes. However, under RDUT, since risk attitude is probability-dependent, a different interpretation is possible but has not been addressed in the transport literature. One exception is de Lapparent and Ben-Akiva (2014) who discuss weak risk aversion only in the dual theory framework of Yaari (1987). Indeed,

\footnotetext{
${ }^{7}$ The Bayesian Information Criterion (BIC) is not reported here but provides the same ranking.

${ }^{8} P\left(\bar{\rho}_{2}^{2}-\bar{\rho}_{1}^{2}>z\right) \leq \Phi\left\{-[-2 N z \ln J+(K 2-K 1)]^{1 / 2}\right\}$, with $\mathrm{N}$ the sample size, J the number of alternatives, $\mathrm{z}$ the observed difference between $\bar{\rho}^{2}, K_{l}$ the number of parameters in Model $1(l=1,2)$, and $\Phi$ the standard normal cumulative distribution function. The table with the upper bound of the p-values of the test is available on request from the authors.

${ }^{9}$ Based on theoretical assumptions, Beaud et al. (2016) consider risk-averse decision makers with a concave value function. They therefore derive the opposite result: a VOT which increases with travel time.
} 
although people are willing to take risks in hopes of having a shorter travel time when faced with two unknown travel times, they may still be weakly risk-averse and prefer average travel time to travel time prospect.

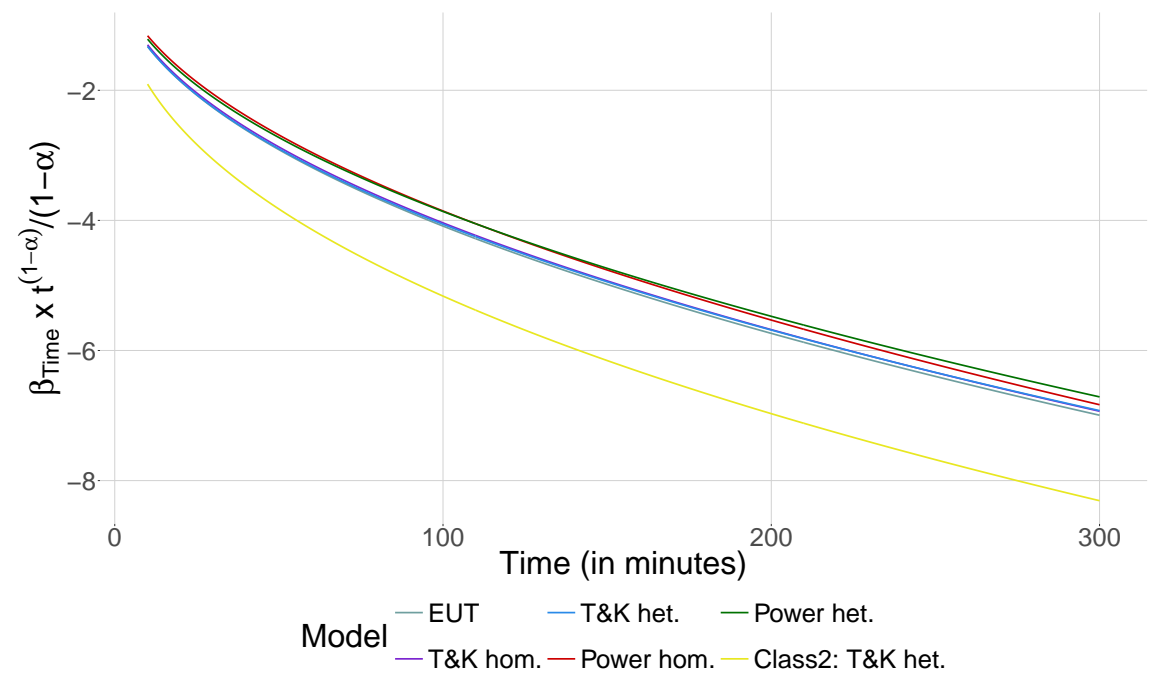

Figure 2 Desutility (in $€$ ) associated with travel time for the six models taking into account a risk aversion parameter.

Notes: hom. $=$ homogeneous weighting function across modes; het. = heterogeneous weighting function across modes

All $\delta$ parameters estimated in Model 3 to 7 are significantly different from one. For Model 3, the coefficient $\delta$ is lower than one, indicating that probabilities are overall overweighted (see Figure 3). People perceive delays as more probable than they are. Considering a power probability weighting function, de Lapparent and Ben-Akiva (2014) find a similar result for commuters.

However, Model 4 reveals a strong heterogeneity across travel modes: probabilities are not perceived the same way for train and for car trips. For car trips, probabilities are underweighted, which supports Hypothesis 1 and, for train trips, probabilities are overweighted, which supports Hypothesis 2.

Model 5 makes it possible to differentiate low and high probability weightings. Since the coefficient $\delta=0.783$ is lower than one, low probabilities are overweighted whereas high probabilities are underweighted, with a threshold ${ }^{10} p^{\star}=0.418$. The results for train trips are consistent with the theory and with previous findings considering a $T \& K$ probability weighting function. In the loss domain, Tversky and Kahneman (1992) estimate $\delta=0.69$. When applied to the transport field, similar values are elicited. For example, Jou and Chen (2013) find that $\delta$ is comprised between 0.36 and 0.66 depending on the trip purpose and the traffic usually encountered on the freeway. Razo and Gao (2013) find a value of 0.55. As in Model 5, these references exhibit $\delta$ coefficients different from one, which shows that EUT does not apply. In the literature, two exceptions are identified. Firstly, de Lapparent and Ben-Akiva (2014) show that for leisure purposed, EUT applies. Secondly, Hensher and Li

\footnotetext{
${ }^{10}$ The value of the threshold is obtained by resolving the equation $w\left(p_{j}\right)=p_{j}$, for $0<p_{j}<1$
} 


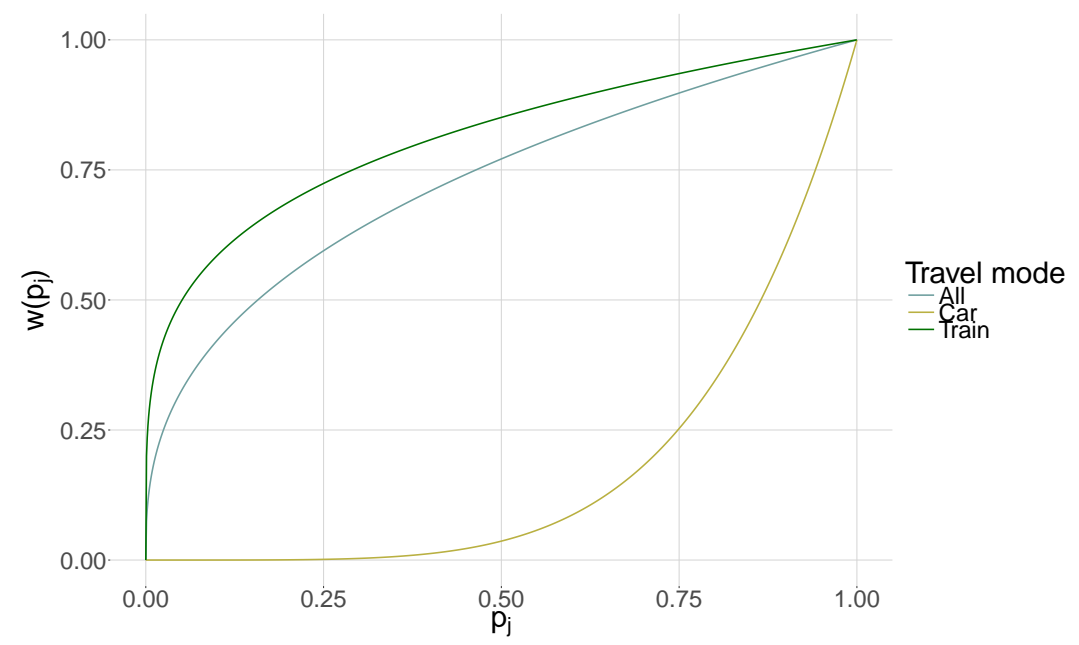

(A) Nested logit, power transformation of the probability weighting function (Models 3 and 4)

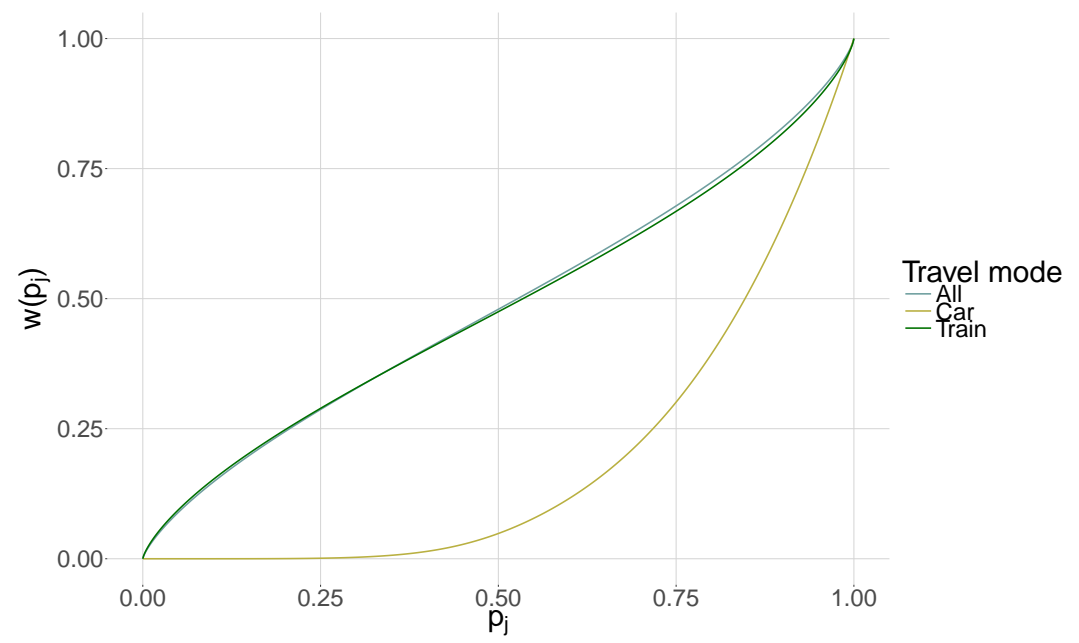

(B) Nested logit, T\&K transformation of the probability weighting function (Models 5 and 6 )

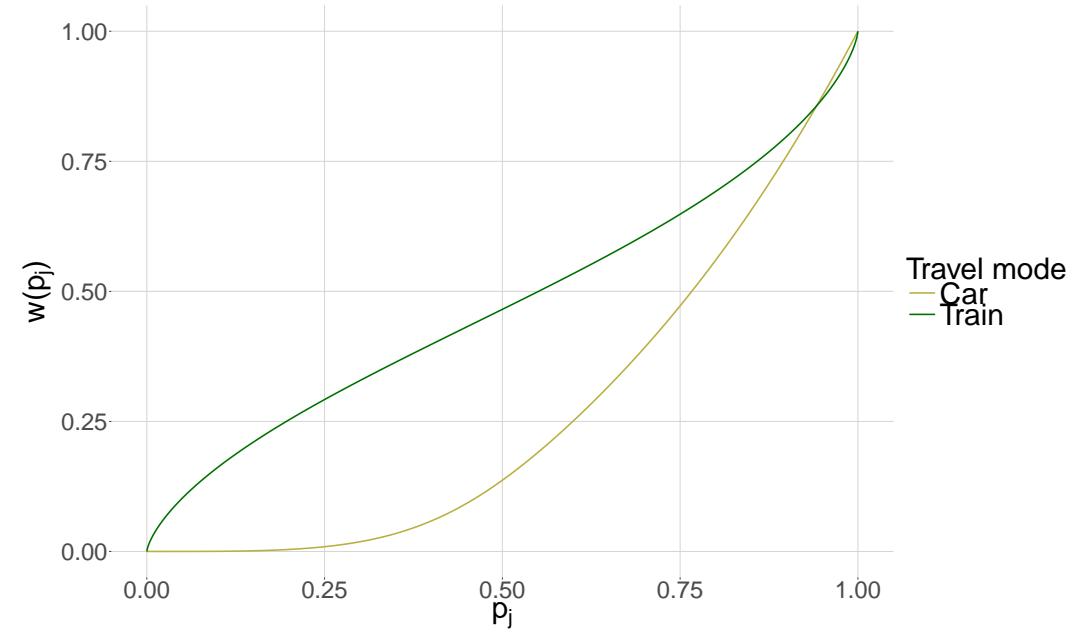

(C) Latent-class model, Class2, T\&K transformation of the probability weighting function (Model 7)

Figure 3 Probability weighting functions for 1 the three models with heterogeneous weights across modes. Notes: $p_{j}$ is the probability of delay associated with the travel mode $j$ $(j \in\{$ Car, Train $\}) ; w\left(p_{j}\right)$ is the weighted probability. 
(2012) estimate $\delta=2.126$ with a multinomial logit and $\delta=5.311$ with a mixed logit model. This result is similar to the one found for car trips in Model 6.

Indeed, Model 6 confirms and refines the heterogeneity unveiled by Model 4. For car trips, there is a clear underweighting of probabilities $\left(\delta^{\text {Train }}=5.17\right)$. For train trips, the same result is found as in the general case but with a somewhat lower coefficient $\left(\delta^{C a r}=0.762\right)$ and, therefore, a lower threshold: $p^{\star}=0.409$. In the database, regular travel times are associated with high probabilities ( 0.8 to 0.95$)$, whereas delays are associated with low probabilities (0.05 to 0.2$)$. This means that the probabilities of delay are overweighted, placing the train mode at a disadvantage in regard to reliability perception.

Model 7 further investigates heterogeneity by defining two classes that behave differently. The probability of belonging to the first class, in which travel time is considered as equal to its expected value, is higher for train users than for car users. Hypothesis 3 is therefore supported by the data: the distinction in the weighting of probabilities is more accurate for car users than for train users who are more likely to consider objective probabilities when making their choice. Train users evaluate travel time prospect accounting only for the corresponding expected travel time. Class 2 accounts for $60 \%$ of the sample, which implies that more than half of respondents do not make their decision according to EUT. In class 2, age and gender do not impact mode choice, since the associated coefficients are not significantly different from zero. The coefficients associated with the travel time variables are not significant, which means that, overall, car users are insensitive to travel time. An explanation relies on the nature of the data: car users tend to stick to their actual mode choice, even if the travel time by train is considerably shorter. For class 2, the probability weighting function indicates that probabilities under 0.394 are overweighted and probabilities above 0.394 are underweighted.

In conclusion, the results indicate that individuals, and especially car users, are riskseekers for car trips. Indeed, the utility function is convex and the probability function is concave. If such a result might seem surprising, it is consistent with Tversky and Kahneman (1992) finding that decision makers are risk-averse in gains but risk-seekers in losses. People would rather choose a travel time that has a $50 \%$ chance of being 60 minutes and a $50 \%$ chance of being 120 minutes than a travel time which has a $100 \%$ chance of being 90 minutes. With regard to uncertain travel time, de Palma and Picard (2005) also find that some travelers (slightly less than one third of their sample) are risk seekers. Concerning train trips, further work is needed to compare the curvatures of the utility and the probability weighting functions.

\subsection{Value of time}

Travel times in a range from 10 to 300 minutes are simulated. Overall, the estimated VOTs are consistent with previous works (Quinet et al., 2014, Wardman et al., 2012; Shires and De Jong, 2009).

For the nested logit models taking into account risk aversion, the mean VOT for car trips is almost $€ 3$ higher than the mean VOT for train trips (see Table 3, lines two to six). Consistent with the finding that the first class of the latent class model is favored by train users, the value for train trips is much lower (€12.1/hour) than the VOT for car trips (€30.4/hour). Conversely, in the second class, the two values are similar (around 
$€ 14.5$ /hour).

For the models including a risk aversion parameter, the diminishing sensitivity of utility to travel time implies a decreasing VOT (see Figure 4). VOTs range from $€ 53 /$ hour for short travel times of 10 minutes to $€ 7.4$ /hour for long travel times of five hours. This result is intuitive: people are ready to pay more to travel for 10 minutes instead of 70 minutes than to travel for 5 hours instead of 6 hours.

Table 3 Value of time (in $€ /$ hour)

\begin{tabular}{lll}
\hline Model & Value of time (train) & Value of time (car) \\
\hline Expected & 12.53 & 13.40 \\
EUT & 12.68 & 15.36 \\
Power hom. & 12.56 & 15.43 \\
Power het. & 12.83 & 15.20 \\
K\&T hom. & 12.56 & 15.25 \\
K\&T het. & 12.63 & 15.41 \\
Class 1 Expected & 12.12 & 30.44 \\
Class 2 K\&T het. & 14.72 & 14.45 \\
\hline
\end{tabular}

Notes: hom.=homogeneous; het.=heterogeneous; EUT: Expected Utility Theory; Power: Power probability weighting function; T\&K: Tversky and Kahneman probability weighting function. The delay probability is set to 0.1 and the delay time is set to 10 minutes. 


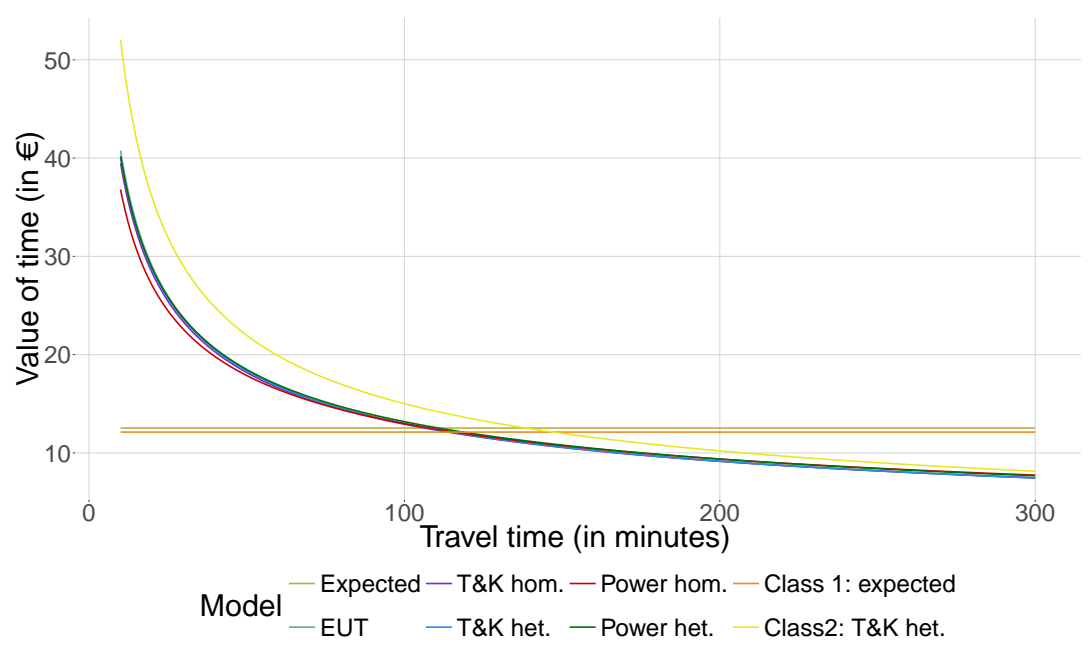

(A) Value of time: train

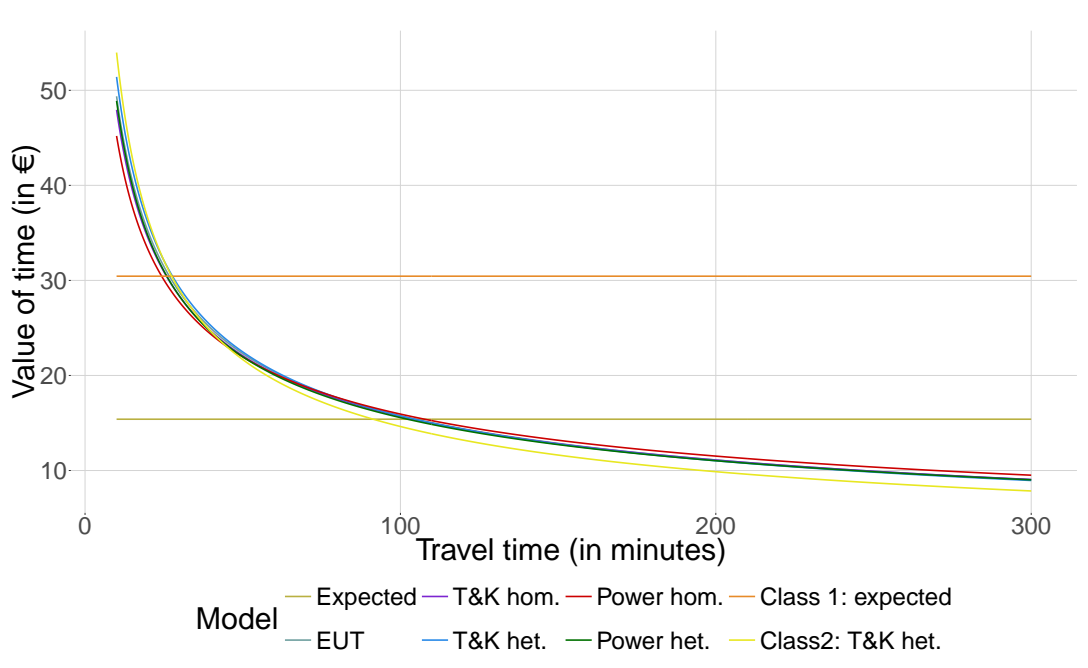

(B) Value of time: car

Figure 4 Value of time associated with the train mode (a) and the car mode (b) for the seven estimated models (including two classes for the latent class model).

Notes: hom. = homogeneous weighting function across modes; het. = heterogeneous weighting function across modes. The delay probability is set to 0.1 and the delay time is set to 10 minutes. 


\section{Conclusion}

Using a choice experiment with choices among train and car modes, this paper investigates the intra- and inter-individual heterogeneity in behavior toward risk. The results highlight the difference of perceptions of probabilities across modes: delay probabilities associated with car trips are under-evaluated, whereas they are over-evaluated for train trips, at least for small probabilities such as those observed in the Rhône-Alpes region. The analysis of inter-individual heterogeneity uncovers two attitudes toward risk and travel time. Some individuals, mainly train users, are risk-neutral and consider the objective expected VOT. Other individuals, mainly car users, are risk-seekers for car trips but do not seem to be sensitive to changes in travel time. This behavior probably results from the use of stated preferences data and needs to be further investigated. The VOTs are consistent with the literature. This work also shows that the VOTs decrease with travel time and are higher for car trips than for train trips, especially for train users.

There are two key directions in which future research might build on the work presented here. Firstly, empirical investigation could consist in a more in-depth investigation of heterogeneity by means of alternative models, such as mixed logit to study the distribution of the relative risk aversion parameter, or the integration of latent variables, such as perceptions of time use or feelings during travel by public transport modes. Using the same method as in this paper, it would also be interesting to test the robustness of the results on other datasets. Secondly, only selective features of cumulative prospect theory have been introduced in the models. Since, the stated preferences survey is personalized with the characteristics of an actual trip, a full integration of cumulative prospect theory (Tversky and Kahneman, 1992) might be considered. The current travel time may be considered as the reference, and the different travel times presented in the choice questions may be framed as gains and losses to study reference dependence and loss aversion. 


\section{References}

Allais, M. (1953). La psychologie de l'homme rationnel devant le risque: la théorie et l'expérience. Journal de la Société de Statistique de Paris, 94:47-73.

Batarce, M. and Ivaldi, M. (2014). Urban travel demand model with endogenous congestion. Transportation Research Part A: Policy and Practice, 59:331-345.

Bates, J., Polak, J., Jones, P., and Cook, A. (2001). The valuation of reliability for personal travel. Transportation Research Part E: Logistics and Transportation Review, 37(2):191229.

Baumeister, R. F., Bratslavsky, E., Finkenauer, C., and Vohs, K. D. (2001). Bad is stronger than good. Review of general psychology, 5(4):323.

Beaud, M., Blayac, T., and Stéphan, M. (2016). The impact of travel time variability and travelers' risk attitudes on the values of time and reliability. Transportation Research Part B: Methodological, 93:207-224.

Ben-Akiva, M. and Swait, J. (1986). The akaike likelihood ratio index. Transportation Science, 20(2):133-136.

Bhat, C. R. and Sardesai, R. (2006). The impact of stop-making and travel time reliability on commute mode choice. Transportation Research Part B: Methodological, 40(9):709-730.

Bierlaire, M. (2016). Pythonbiogeme: a short introduction. Technical report, Transp-OR, Ecole Polytechnique Federale de Lausanne.

Börjesson, M., Eliasson, J., and Franklin, J. P. (2012). Valuations of travel time variability in scheduling versus mean-variance models. Transportation Research Part B: Methodological, 46(7):855-873.

Cohen, M. (2015). Risk perception, risk attitude, and decision: a rank-dependent analysis. Mathematical Population Studies, 22(1):53-70.

de Lapparent, M. and Ben-Akiva, M. (2014). Risk aversion in travel mode choice with rank-dependent utility. Mathematical Population Studies, 21(4):189-204.

de Palma, A., Ben-Akiva, M., Brownstone, D., Holt, C., Magnac, T., McFadden, D., Moffatt, P., Picard, N., Train, K., Wakker, P., et al. (2008). Risk, uncertainty and discrete choice models. Marketing Letters, 19(3-4):269-285.

de Palma, A. and Picard, N. (2005). Route choice decision under travel time uncertainty. Transportation Research Part A: Policy and Practice, 39(4):295-324.

de Palma, A., Picard, N., and de Lapparent, M. (2015). Risk and uncertainty in urban and transport economics. Mathematical Population Studies, 22(1):1-3.

Denant-Boèmont, L. and L'Haridon, O. (2013). La rationalité à l'épreuve de l'économie comportementale. Revue française d'économie, 28(2):35-89.

Diecidue, E. and Wakker, P. P. (2001). On the intuition of rank-dependent utility. Journal of Risk and Uncertainty, 23(3):281-298.

Fox, C. R. and Tversky, A. (1995). Ambiguity aversion and comparative ignorance. The quarterly journal of economics, 110(3):585-603.

Greene, W. H. and Hensher, D. A. (2003). A latent class model for discrete choice analysis: contrasts with mixed logit. Transportation Research Part B: Methodological, 37(8):681698.

Hensher, D., Rose, J., and Greene, W. (2015). Applied Choice Analysis. Number 1188. Cambridge University Press. 
Hensher, D. A., Greene, W. H., and Li, Z. (2011). Embedding risk attitude and decision weights in non-linear logit to accommodate time variability in the value of expected travel time savings. Transportation research part B: methodological, 45(7):954-972.

Hensher, D. A. and Li, Z. (2012). Valuing travel time variability within a rank-dependent utility framework and an investigation of unobserved taste heterogeneity. Journal of Transport Economics and Policy, pages 293-312.

Hjorth, K. and Fosgerau, M. (2012). Using prospect theory to investigate the low marginal value of travel time for small time changes. Transportation Research Part B: Methodological, 46(8):917-932.

Holt, C. A., Laury, S. K., et al. (2002). Risk aversion and incentive effects. American economic review, 92(5):1644-1655.

Jou, R.-C. and Chen, K.-H. (2013). An application of cumulative prospect theory to freeway drivers' route choice behaviours. Transportation Research Part A: Policy and Practice, 49:123-131.

Kahneman, D. and Tversky, A. (1979). Prospect theory: An analysis of decision under risk. Econometrica: Journal of the Econometric Society, pages 263-291.

Kemel, E. and Paraschiv, C. (2013). Prospect theory for joint time and money consequences in risk and ambiguity. Transportation Research Part B: Methodological, 56:81-95.

König, A. and Axhausen, K. W. (2002). The reliability of the transportation system and its influence on the choice behaviour. In Swiss Transport Research Conference.

Li, Z. and Hensher, D. (2011). Prospect theoretic contributions in understanding traveller behaviour: a review and some comments. Transport reviews, 31(1):97-115.

Mazar, N., Koszegi, B., and Ariely, D. (2014). True context-dependent preferences? the causes of market-dependent valuations. Journal of Behavioral Decision Making, 27(3):200208.

Palmier, P. (2010). Mode d'emploi - musliw - logiciel de calcul d'accessibilité multimodal. Technical report, CETE Nord-Picardie.

Prelec, D. (1998). The probability weighting function. Econometrica, pages 497-527.

Quiggin, J. (1982). A theory of anticipated utility. Journal of Economic Behavior \& Organization, 3(4):323-343.

Quinet, E. et al. (2014). L'évaluation socioéconomique des investissements publics. Technical report, HAL.

Rasouli, S. and Timmermans, H. (2014). Applications of theories and models of choice and decision-making under conditions of uncertainty in travel behavior research. Travel Behaviour and Society, 1(3):79-90.

Razo, M. and Gao, S. (2013). A rank-dependent expected utility model for strategic route choice with stated preference data. Transportation Research Part C: Emerging Technologies, 27:117-130.

Rose, J. M. and Bliemer, M. C. (2007). Stated preference experimental design strategies. In Handbook of Transport Modelling: 2nd Edition, pages 151-180. Emerald Group Publishing Limited.

Senbil, M. and Kitamura, R. (2005). Heterogeneity in commuter departure time decision: A prospect theoretic approach. In Applied Research in Uncertainty Modeling and Analysis, pages 369-398. Springer.

Shires, J. D. and De Jong, G. C. (2009). An international meta-analysis of values of travel 
time savings. Evaluation and program planning, 32(4):315-325.

Sun, Z., Arentze, T., and Timmermans, H. (2012). A heterogeneous latent class model of activity rescheduling, route choice and information acquisition decisions under multiple uncertain events. Transportation research part C: emerging technologies, 25:46-60.

Timmermans, H. (2010). On the (ir) relevance of prospect theory in modelling uncertainty in travel decisions. EJTIR, 4(10):368-384.

Train, K. E. (2009). Discrete choice methods with simulation. Cambridge university press.

Tversky, A. and Fox, C. R. (1995). Weighing risk and uncertainty. Psychological review, 102(2):269.

Tversky, A. and Kahneman, D. (1992). Advances in prospect theory: Cumulative representation of uncertainty. Journal of Risk and uncertainty, 5(4):297-323.

Tversky, A. and Simonson, I. (1993). Context-dependent preferences. Management science, 39(10):1179-1189.

Wardman, M., Chintakayala, P., de Jong, G., and Ferrer, D. (2012). European wide metaanalysis of values of travel time. Technical report, ITS, University of Leeds, Paper prepared for EIB.

Wu, G. and Gonzalez, R. (1999). Nonlinear decision weights in choice under uncertainty. Management science, 45(1):74-85.

Xiao, Y. and Fukuda, D. (2015). On the cost of misperceived travel time variability. Transportation Research Part A: Policy and Practice, 75:96-112.

Yaari, M. E. (1987). The dual theory of choice under risk. Econometrica: Journal of the Econometric Society, pages 95-115. 